\title{
Early N-terminal pro-B-type natriuretic peptide is associated with cardiac complications and function during pregnancy in congenital heart disease
}

\author{
A. S. Siegmund · P. G. Pieper · B. J. Bouma · F. M. Rosenberg · H. Groen · C. M. Bilardo · D. J. van Veldhuisen · \\ M. G. Dickinson
}

Accepted: 14 January 2021 / Published online: 3 February 2021

(C) The Author(s) 2021

\begin{abstract}
Background Elevated $N$-terminal pro-B-type natriuretic peptide (NT-proBNP) levels at 20 weeks' gestation predict adverse cardiovascular (CV) complications during pregnancy in women with congenital heart disease (CHD). To improve early risk assessment in these women, we investigated the predictive value of first-trimester NT-proBNP for CV complications and its association with ventricular function during pregnancy.

Methods Pregnant women with CHD, previously enrolled in a prospective national study or evaluated by an identical protocol, were included. Clinical data, echocardiographic evaluation and NT-proBNP measurements were obtained at 12, 20 and 32 weeks' gestation. Elevated NT-proBNP was defined as $>235 \mathrm{pg} / \mathrm{ml}$ (95th percentile reference value of healthy pregnant women in the literature).
\end{abstract}

A. S. Siegmund · P. G. Pieper · F. M. Rosenberg ·

D. J. van Veldhuisen · M. G. Dickinson $(\varangle)$

Department of Cardiology, University Medical Centre

Groningen, University of Groningen, Groningen, The Netherlands

m.g.dickinson@umcg.nl

\section{B. J. Bouma}

Department of Cardiology, Heart Centre, Amsterdam UMC, Amsterdam Cardiovascular Sciences, University of Amsterdam, Amsterdam, The Netherlands

\section{H. Groen}

Department of Epidemiology, University Medical Centre Groningen, University of Groningen, Groningen, The Netherlands

\section{M. Bilardo}

Department of Obstetrics, University Medical Centre Groningen, University of Groningen, Groningen, The Netherlands
Results We examined 126 females (mean age 29 years). Elevated NT-proBNP at 12 weeks was associated with CV complications ( $n=7,5.6 \%$, odds ratio 10.9 , $p=0.004$ ). Arrhythmias were the most common complication $(71 \%)$. The negative predictive value of low NT-proBNP to exclude CV complications was $97.2 \%$. In women with CV complications, NT-proBNP levels remained high throughout pregnancy, while a decrease was seen in women without CV complications ( $p<0.001$ for interaction between group and time). At 12 weeks, higher NT-proBNP levels were associated with impaired subpulmonary ventricular function $(p<0.001)$ and also with a decline in subpulmonary ventricular function later in pregnancy $(p=0.012)$. Conclusions In this study, first-trimester NT-proBNP levels were associated with adverse $\mathrm{CV}$ complications and a decline in subpulmonary ventricular function later in pregnancy in women with CHD. Early NT- 
proBNP evaluation is useful for tailored care in pregnant women with CHD.

Keywords Congenital heart disease $\cdot \mathrm{N}$-terminal pro-B-type natriuretic peptide · Pregnancy • Cardiovascular complications · Right ventricular function

\section{Introduction}

In women with congenital heart disease (CHD), several prediction models are available to estimate the risk of maternal cardiovascular (CV) complications during pregnancy [1-3]. However, early prediction of these risks remains challenging. We have previously shown that elevated $N$-terminal pro-B-type natriuretic peptide (NT-proBNP) levels at 20 weeks' gestation are an independent risk predictor for $\mathrm{CV}$ complications in pregnant women with CHD [4]. No data are available about the prognostic value of NTproBNP in early pregnancy or the (normal) course of NT-proBNP during pregnancy in women with CHD. NT-proBNP is primarily secreted when abnormal ventricular wall stress and volume overload occur, both of which can occur in early pregnancy $[5,6]$. From a pathophysiological point of view, early NT-proBNP detection makes sense in CHD patients. Many women with (corrected) CHD start pregnancy with impaired cardiac function, and it is likely that haemodynamic changes during pregnancy expose them to the risk of cardiac maladaptation from the first trimester. Moreover, pregnancy can be associated with persisting structural cardiac remodelling, recurring arrhythmias and deterioration in ventricular function (in particular right/subpulmonary ventricular function) [7-10]. Subpulmonary ventricular dysfunction is an independent predictor for CV complications in pregnant women with CHD [4] and is known to be correlated with elevated NT-proBNP in non-pregnant patients $[11,12]$. Identification of impaired cardiac function as early as possible might be effective in preventing CV complications later in pregnancy.

To improve early risk assessment in pregnant women with $\mathrm{CHD}$, we aimed to investigate the predictive value of first-trimester NT-proBNP for CV complications later in pregnancy and its association with ventricular function during pregnancy.

\section{Methods}

\section{Study design}

This present study comprised pregnant women enrolled in the prospective multicentre observational cohort ZAHARA III study (Zwangerschap bij Aangeboren HARtAfwijkingen, pregnancy in congenital heart disease) or evaluated by an identical protocol (during standard patient care in our university medical centre). The study design of the ZAHARA III study has been reported previously [13]. Pregnant women with CHD, aged $\geq 18$ years, presenting in one of the participating centres at $\leq 14$ weeks' gestation and with NTproBNP measurements available ( $\leq 14$ weeks' gestation) were eligible for enrolment in the current study. All the participating centres received the approval of the medical ethics committee and all women (prospectively enrolled) provided written informed consent.

\section{Pregnancy follow-up and CV complications}

At the first ante-partum visit, preconception baseline characteristics of pregnant women were recorded, including: underlying heart disease, CV and obstetric history, age, cardiac status (including New York Heart Association functional class, modified World Health Organisation risk class for maternal risk of $\mathrm{CV}$ complications [3], electrocardiogram, laboratory results and echocardiographic recordings), medication use and intoxications. Evaluation of pregnancy was performed at 12, 20 and 32 weeks, including clinical evaluation, standardised echocardiogram, electrocardiogram and laboratory evaluation. All echocardiograms were performed according to an identical ZAHARA study protocol [14], and chamber quantification, valvular function, and systolic and diastolic ventricular function were assessed according to the current guidelines [15-17]. Systemic ventricular dysfunction was defined as ejection fraction $<45 \%$ and subpulmonary ventricular dysfunction was defined as tricuspid annular plane systolic excursion (TAPSE) $<17 \mathrm{~mm}$. Consistency and accuracy of the echocardiography data were checked by A.S.S. NT-proBNP at 12 weeks was considered abnormal if the value exceeded the 95th percentile reference values according to gestational age in healthy pregnant women (>235 ng/ml) [18].

CV complications were evaluated during pregnancy and up to 6 months post-partum. Primary CV complications were defined as the need for an urgent invasive $\mathrm{CV}$ procedure, heart failure (according to the guidelines and documented by the attending physician [19]), new-onset or symptomatic tachy- or bradyarrhythmia requiring new or extended treatment, thromboembolic events, myocardial infarction, cardiac arrest, cardiac death, endocarditis and aortic dissection.

\section{Statistical analysis}

Data are presented as mean \pm standard deviation, median (25th to 75 th percentiles) or numbers (percentages). For the comparison of dichotomous variables, we used the $\chi^{2}$ test or Fisher exact test, as appropriate. Univariable logistic regression was used to assess associations between predefined preconception cardiac function parameters, elevated NT-proBNP at 12 weeks and primary $\mathrm{CV}$ complications. 
Table 1 Demographics and clinical data

\begin{tabular}{|c|c|}
\hline & $n=126$ \\
\hline \multicolumn{2}{|l|}{ Primary CHD } \\
\hline Left-sided lesions & $41(32.5)$ \\
\hline - Repaired aortic coarctation & $18(14.3)$ \\
\hline - Aortic valve stenosis/bicuspid aortic valve & $18(14.3)$ \\
\hline - Mitral valve disease & $5(4.0)$ \\
\hline Right-sided lesions & $34(27.0)$ \\
\hline - Double-chambered right ventricle & $1(0.8)$ \\
\hline - Ebstein's anomaly & $5(4.0)$ \\
\hline - Pulmonary atresia & $1(0.8)$ \\
\hline - Pulmonary valve stenosis & $14(11.1)$ \\
\hline - Tetralogy of Fallot & $13(10.3)$ \\
\hline Shunt lesions & $36(28.6)$ \\
\hline - Abnormal pulmonary venous return & $1(0.8)$ \\
\hline - Atrial septal defect & $10(7.9)$ \\
\hline - Atrioventricular septal defect & $7(5.6)$ \\
\hline - Ventricular septal defect & $18(14.3)$ \\
\hline Connective tissue disorder & $3(2.4)$ \\
\hline Right aortic arch & $1(0.8)$ \\
\hline Complex CHD & $11(8.7)$ \\
\hline - Fontan circulation & $1(0.8)$ \\
\hline $\begin{array}{l}\text { - Transposition of great arteries (Mustard/Senning correc- } \\
\text { tion) }\end{array}$ & $3(2.4)$ \\
\hline - Transposition of great arteries (arterial switch correction) & $3(2.4)$ \\
\hline - Congenitally corrected transposition of great arteries & $2(1.6)$ \\
\hline $\begin{array}{l}\text { - Right ventricular hypoplasia with pulmonary valve steno- } \\
\text { sis and bilateral Glenn procedure }\end{array}$ & $1(0.8)$ \\
\hline - Truncus arteriosus & $1(0.8)$ \\
\hline \multicolumn{2}{|l|}{ NYHA class } \\
\hline-1 & $111(88.1)$ \\
\hline$-\|$ & $9(7.1)$ \\
\hline \multicolumn{2}{|l|}{ Modified WHO class $^{\mathrm{a}}$} \\
\hline-1 & $16(12.7)$ \\
\hline$-\|$ & $87(69.0)$ \\
\hline- III & $23(18.3)$ \\
\hline Mechanical valve prosthesis & $7(5.6)$ \\
\hline Prior cardiac event (HF, TIA, stroke, arrhythmia) & $19(15.1)$ \\
\hline Hypertension before pregnancy & $5(6.1)$ \\
\hline Pacemaker & $2(1.6)$ \\
\hline Cardiac medication use prior to pregnancy ${ }^{\mathrm{a}}$ & $28(30.1)$ \\
\hline - Beta-blocker & $21(22.6)$ \\
\hline - Other & $13(14.0)$ \\
\hline \multicolumn{2}{|l|}{ Echocardiographic parameters ${ }^{\mathrm{a}, \mathrm{b}}$} \\
\hline Systemic AV valve regurgitation ${ }^{c}$ & $3(3.7)$ \\
\hline Pulmonary AV valve regurgitation ${ }^{c}$ & $6(7.3)$ \\
\hline Pulmonary stenosis $^{d}$ & $5(6.1)$ \\
\hline Pulmonary valve regurgitation ${ }^{c}$ & $12(14.6)$ \\
\hline Aortic valve stenosis $^{d}$ & $7(8.5)$ \\
\hline
\end{tabular}

Table 1 (Continued)

\begin{tabular}{|c|c|}
\hline & $=126$ \\
\hline Aortic valve regurgitation ${ }^{c}$ & $2(2.4)$ \\
\hline Systemic ventricular systolic dysfunction ${ }^{\mathrm{e}}$ & $3(3.7)$ \\
\hline Systemic ventricular hypertrophy ${ }^{\dagger}$ & $6(7.3)$ \\
\hline Subpulmonary ventricular systolic dysfunction ${ }^{g}$ & 17 (20.7) \\
\hline \multicolumn{2}{|c|}{$\begin{array}{l}\text { AV atrioventricular, CHD congenital heart disease, HF heart failure, } \\
\text { NYHA New York Heart Association, TAPSE tricuspid annular plane systolic } \\
\text { excursion, } T I A \text { transient ischaemic attack, WHO World Health Organisation } \\
\text { Data are reported as } n(\%) \\
\text { a } \leq 1 \text { year before pregnancy } \\
\text { bAvailable in } 82(65.1 \%) \text { women } \\
\text { 'Moderate or severe regurgitation } \\
\text { dPeak gradient } \geq 36 \mathrm{mmHg} \\
\text { eEjection fraction }<45 \% \\
\text { 'Systemic ventricular mass/body surface area }>95 \mathrm{~g} / \mathrm{m}^{2} \\
{ }^{2} \text { TAPSE }<17 \mathrm{~mm}\end{array}$} \\
\hline
\end{tabular}

NT-proBNP levels during pregnancy were logtransformed to create a normal distribution for further statistical analyses. Uni- and multivariable linear regression models were used for associations between NT-proBNP and cardiac function parameters based on the literature [20]. Variables associated with the studied end points $(p<0.10)$ were entered in the multivariable model. The final model was constructed by backward deletion. Women with a systemic right ventricle were excluded from analyses with subpulmonary ventricular function.

For longitudinal analyses (at 12, 20 and 32 weeks), analyses using generalised estimating equations (GEE) were performed with an unstructured correlation matrix, and data are presented as mean with standard error. For longitudinal comparisons within groups (women with vs without CV complications), an interaction (time $\times$ group) was included in the model. Statistical analyses were performed using SPSS version 23.0 (SPSS, Chicago, IL, USA).

\section{Results}

\section{Baseline characteristics}

In the ZAHARAIII study, 204 pregnant women were included initially. Eleven women were excluded, because of a miscarriage $(n=6)$, absence of CHD $(n=4)$ or withdrawal of informed consent $(n=1)$. In the current study, only pregnant women with NTproBNP $\leq 14$ weeks available were included, resulting in a study population of 126 pregnant women. Underlying CHD and baseline characteristics are presented in Tab. 1. Maternal age at conception was $28.8 \pm 4.2$ years, body mass index was $23.8 \pm 3.9 \mathrm{~kg} / \mathrm{m}^{2}$, and $62(49.2 \%)$ women were nulliparous. Prior CV events were reported in 19 (15.1\%) women. Eighteen women had a history of arrhythmia and one woman had a history of transient ischaemic attack. 


\section{CV complications and first-trimester NT-proBNP}

CV complications were observed in 7 (5.6\%) women. Five $(71.4 \%)$ women had arrhythmias (mainly supraventricular tachyarrhythmias), for which cardioversion and/or increased metoprolol dosage was required. Underlying heart diseases were: mitral valve disease, aortic valve stenosis, transposition of great arteries (Mustard/Senning correction) and Fontan circulation. Arrhythmias occurred between 12 and 28 weeks of pregnancy, except for one woman who had arrhythmia 3 months post-partum. Two (28.6\%) women had thromboembolic complications for which anticoagulation therapy was required; one woman (with Mustard correction) had deep vein thrombosis at 23 weeks; and one woman (with connective tissue disorder) had arterial thrombosis at 38 weeks. NTproBNP was measured at 11.5 (10-13) weeks. Women with CV complications had higher NT-proBNP at 12 weeks compared with women without complications, 301 (117-381) $\mathrm{ng} / \mathrm{ml}$ versus 95 (53-170) $\mathrm{ng} / \mathrm{ml}$ $(p=0.008)$. Four women $(57.1 \%)$ with CV complications had NT-proBNP $>235 \mathrm{ng} / \mathrm{ml}$. Of all women, $86.5 \%$ had NT-proBNP $\leq 235 \mathrm{ng} / \mathrm{ml}(89.1 \%$ of women without CV complications). NT-proBNP $\leq 235 \mathrm{ng} / \mathrm{ml}$ at 12 weeks had a negative predictive value of $97.2 \%$

Table 2 Logistic regression analysis of preconception values, elevated $N$-terminal pro-B-type natriuretic peptide (NT-proBNP) in first trimester and cardiovascular complications during pregnancy

\begin{tabular}{|c|c|c|c|}
\hline Variables & $\mathrm{OR}$ & $95 \% \mathrm{Cl}$ & $p$-value \\
\hline \multicolumn{4}{|l|}{ Univariable predictor } \\
\hline Maternal age at conception & 0.9 & $0.8-1.1$ & 0.401 \\
\hline WHO class III or IV & 14.0 & $2.5-77.9$ & 0.003 \\
\hline Parity & 0.4 & $0.1-2.0$ & 0.243 \\
\hline Smoking $^{\mathrm{a}}$ & 2.7 & $0.3-27.6$ & 0.407 \\
\hline Resting heart rate ${ }^{a}$ & 1.0 & $0.9-1.1$ & 0.979 \\
\hline Sinus rhythm ${ }^{a}$ & 0.4 & $0.1-1.7$ & 0.202 \\
\hline Use of cardiac medication ${ }^{\mathrm{a}}$ & 3.2 & $0.7-15.4$ & 0.140 \\
\hline TabelPrior cardiac event & 4.8 & 1.0-23.6 & 0.052 \\
\hline History of arrhythmia & 5.2 & $1.1-25.5$ & 0.042 \\
\hline Mechanical valve prosthesis & 9.1 & $1.4-59.1$ & 0.020 \\
\hline Right systemic ventricle & 15.5 & $2.1-114.4$ & 0.007 \\
\hline Aortic valve stenosis ${ }^{a}$ & 5.6 & $0.9-36.5$ & 0.072 \\
\hline Pulmonary valve stenosis ${ }^{\mathrm{a}}$ & 2.9 & $0.3-30.4$ & 0.371 \\
\hline Aortic valve regurgitation ${ }^{\mathrm{a}}$ & -18.8 & $0-0$ & 0.999 \\
\hline Pulmonary valve regurgitation ${ }^{\mathrm{a}}$ & -19.0 & $0-0$ & 0.999 \\
\hline LVEF $^{\mathrm{a}}$ & 1.0 & $0.9-1.2$ & 0.780 \\
\hline TAPSE $^{\mathrm{a}}$ & 0.8 & $0.7-1.0$ & 0.100 \\
\hline 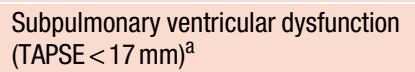 & 4.9 & $0.6-37.7$ & 0.124 \\
\hline Elevated NT-proBNP in first trimester ${ }^{b}$ & 10.9 & $2.2-54.1$ & 0.004 \\
\hline \multicolumn{4}{|c|}{$\begin{array}{l}\text { Cl confidence interval, LVEF systemic ventricular ejection fraction, NYHA New } \\
\text { York Heart Association, OR odds ratio, TAPSE tricuspid annular plane systolic } \\
\text { excursion, WHO World Health Organisation } \\
\text { a } \leq 1 \text { year before pregnancy } \\
\text { bNT-proBNP }>235 \mathrm{pg} / \mathrm{ml}\end{array}$} \\
\hline
\end{tabular}

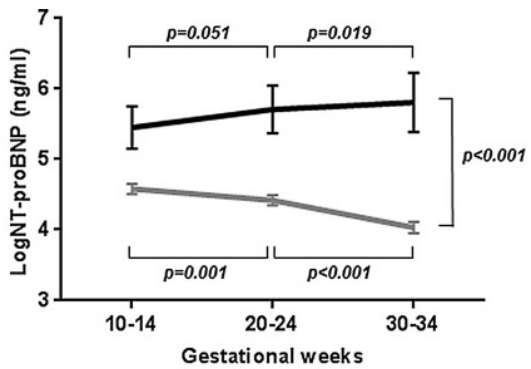

\begin{tabular}{l|lll} 
NT-proBNP in CV complication group & $301[117-381]$ & $492[208-660]$ & $447[50-617]$ a \\
\hline NT-proBNP in no CV complication group & $95[53-170]$ & $96[50-152]$ & $56[35-93]$
\end{tabular}

Fig. 1 Serial analyses of log-transformed $N$-terminal proB-type natriuretic peptide (logNT-proBNP) levels throughout pregnancy in women with cardiovascular (CV) complications (black line, $n=7$ ) and without CV complications (grey line, $n=119$ ). Absolute NT-proBNP levels at 10-14, 20-24 and 30-34 weeks are reported as median (Q1-Q3). aReported as median (minimum-maximum). A significant difference in the course of logNT-proBNP was found between women with CV complications and without CV complications $(p<0.001)$. NT-proBNP levels decreased after 10-14 weeks' gestation in women without CV complications and remained high in women with CV complications

for the occurrence of CV complications after 12 weeks and a positive predictive value of $23.5 \%$. The sensitivity of NT-proBNP $>235 \mathrm{ng} / \mathrm{ml}$ was $57.1 \%$ and the specificity was $89.1 \%$. In Tab. 2 , univariable regression analyses for the identification of predictors for $\mathrm{CV}$ complications during pregnancy are presented.

Fig. 1 presents the course of NT-proBNP throughout pregnancy of women with and without CV complications based on GEE analyses of log-transformed values, corrected for correlation within subjects. An overall difference in the course of NT-proBNP was found between women with and without CV complications $(p<0.001$ for interaction between group and time). More specifically, in women without CV complications NT-proBNP decreased during pregnancy but not in women with $\mathrm{CV}$ complications. In women with $\mathrm{CV}$ complications, NT-proBNP increased between 20-24 and $30-34$ weeks $(p=0.019)$.

\section{Cardiac function and first-trimester NT-proBNP}

All correlations between cardiac function and NTproBNP at 12 weeks are presented in Tab. 3. With multivariable analyses, higher maternal age and reduced subpulmonary systolic ventricular function (TAPSE) remained associated with higher NT-proBNP ( $p=0.004$ and $p<0.001$, respectively). Fig. 2 presents the patterns of TAPSE of women with elevated and normal NT-proBNP at 12 weeks. A difference in the pattern of TAPSE was found ( $p=0.019$ for interaction between group and time). In women with normal NT-proBNP, TAPSE increased between 10-14 weeks' and $20-24$ weeks' gestation $(22.3 \pm 0.5$ to $24.1 \pm 0.6$, 


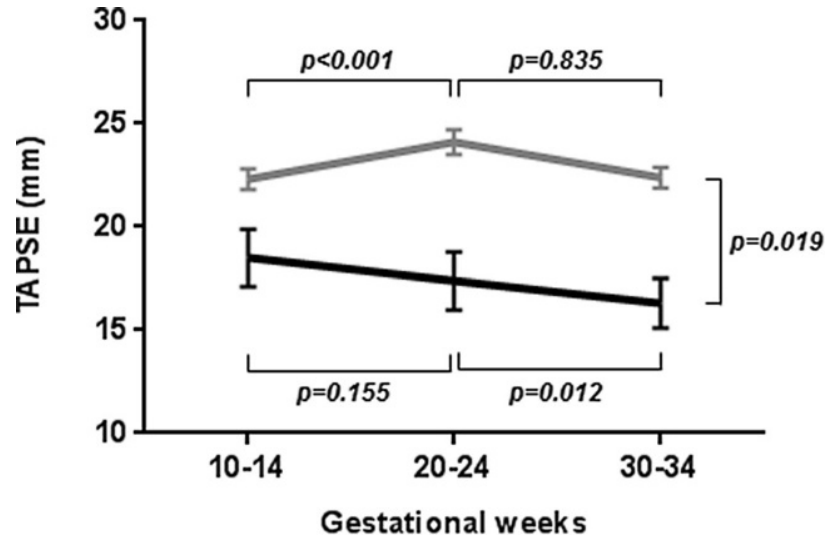

Fig. 2 Patterns of tricuspid annular plane systolic excursion (TAPSE) during pregnancy in women with elevated first-trimester $N$-terminal pro-B-type natriuretic peptide (NTproBNP) levels (black line, $n=12$ ) and women with normal first-trimester NT-proBNP levels (grey line, $n=92$ ). A significant difference in the pattern of TAPSE was found between women with elevated NT-proBNP and those with normal NTproBNP $(p=0.019)$. TAPSE increased from $10-14$ weeks to 20-24 weeks in women with normal NT-proBNP. Only in women with elevated NT-proBNP did TAPSE significantly decrease from 20-24 weeks' to 30-34 weeks' gestation

Table 3 Associations between log-transformed N-terminal pro-B-type natriuretic peptide, age and cardiac function at first trimester

\begin{tabular}{l|c|c|r|r|}
\hline & $n$ & Beta & \multicolumn{1}{l}{$95 \% \mathrm{Cl}$} & $p$-value \\
\hline Age & 126 & 0.044 & $0.013-0.075$ & 0.005 \\
\hline NYHA class & 126 & 0.379 & $0.041-0.718$ & 0.028 \\
\hline LVEDD/BSA & 99 & 0.044 & $-0.008-0.096$ & 0.100 \\
\hline LVESD/BSA & 92 & 0.033 & $-0.016-0.083$ & 0.187 \\
\hline LAVi & 50 & 0.023 & $-0.010-0.055$ & 0.163 \\
\hline LVEF & 103 & -0.037 & $-0.059-0.016$ & 0.001 \\
\hline TAPSE $^{\mathrm{a}}$ & 104 & -0.054 & $-0.080-0.029$ & $<0.001$ \\
\hline S' RV $^{\mathrm{a}}$ & 74 & -0.059 & $-0.113-0.005$ & 0.034 \\
\hline E/A ratio & 86 & 0.286 & $-0.069-0.641$ & 0.113 \\
\hline E' & 75 & 0.021 & $-0.042-0.084$ & 0.515 \\
\hline Multivariable regression analyses & & & \\
\hline Age & 126 & 0.054 & $0.018-0.089$ & 0.004 \\
\hline TAPSE & 104 & -0.060 & $-0.087-0.033$ & $<0.001$
\end{tabular}

$B S A$ body surface area, $C /$ confidence interval, $E$ 'early diastolic tissue Doppler velocity of systemic ventricular annular ring, E/A ratio early to atrial mitral inflow velocity ratio, LAVi indexed systemic atrial volume, LVEDD systemic ventricular end-diastolic diameter, LVEF systemic ventricular ejection fraction, LVESV systemic ventricular end-diastolic diameter, NYHA New York Heart Association, $S$ ' systolic tissue Doppler velocity of subpulmonary ventricular annular ring, TAPSE tricuspid annular plane systolic excursion

${ }^{a}$ Women with systemic right ventricle excluded from analyses

${ }^{\mathrm{b}}$ Degrees of freedom $=72$

$p<0.001)$ but not in women with elevated NT-proBNP $(18.5 \pm 1.4$ to $17.4 \pm 1.4, p=0.155)$. Only in women with elevated NT-proBNP did TAPSE decrease from 20-24 weeks' to $30-34$ weeks' gestation $(17.4 \pm 1.4$ to $16.3 \pm 1.2, p=0.012$ ).
At 12 weeks, 5 (4.0\%) women had systemic ventricular dysfunction and 17 (14.0\%) women had subpulmonary ventricular dysfunction.

\section{Discussion}

This study focuses on the predictive role of firsttrimester NT-proBNP for CV complications later in pregnancy in women with CHD. The main findings are that elevated first-trimester NT-proBNP is associated with adverse CV complications and with a decline in subpulmonary ventricular function during pregnancy. Furthermore, in women with $\mathrm{CV}$ complications NT-proBNP remains high throughout pregnancy. This finding is in contrast to that in women without CV complications, in whom NTproBNP decreases steadily from the second trimester onwards.

Screening for CV complications early in pregnancy makes sense from a pathophysiological point of view, since plasma volume and cardiac output increase, and $75 \%$ of these increases occur in the first trimester [21]. We show for the first time that early NT-proBNP evaluation can be useful in risk estimation in pregnant women with CHD. Low first-trimester NT-proBNP seems to indicate that these women have a good chance of adapting to haemodynamic changes during pregnancy and to complete their pregnancy without CV complications. When first-trimester NT-proBNP is elevated, our findings suggest that these women are at higher risk for $\mathrm{CV}$ complications in pregnancy. In this study, no heart failure complications occurred, and it is therefore important to underline that elevated NTproBNP was associated mainly with the occurrence of arrhythmias. A possible explanation could be that heart failure diagnosis was underreported and/or managed temporarily with extra diuretics before fulminant heart failure symptoms could occur. This could be the result of the stricter guideline-driven peri-pregnancy management we strive for in our centres [3]. The association between NT-proBNP and arrhythmias might be explained by the occurrence of volume overload and wall stress during pregnancy, both leading to increased secretion of NT-proBNP and resulting in a trigger for arrhythmias [22]. By identifying women at risk as early as possible in pregnancy, appropriate follow-up visits, possible treatment of arrhythmias or even prevention of such complications could be managed early on and could lead to a decrease in morbidity. In this study, elevated firsttrimester NT-proBNP was also present in women without CV complications and was associated with impaired subpulmonary ventricular function. The finding that higher NT-proBNP is associated with worse subpulmonary ventricular function is in line with previous studies conducted in non-pregnant patients with right heart disease $[11,12]$. The current results suggest that the subpulmonary ventricle is less well suited to cope with the increased overload 


\section{Advertisement placed here.}

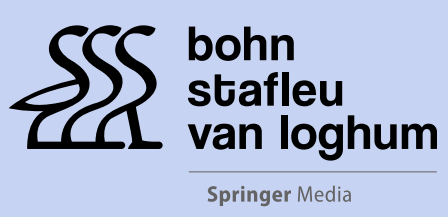

Houten 2021 


\section{Advertisement placed here.}

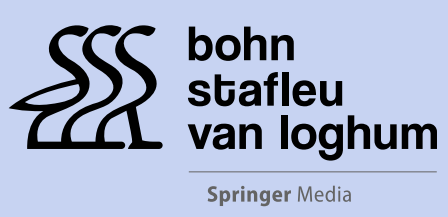

Houten 2021 


\section{Advertisement placed here.}

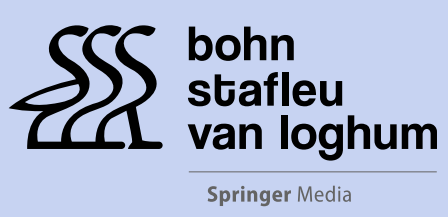

Houten 2021 


\section{Advertisement placed here.}

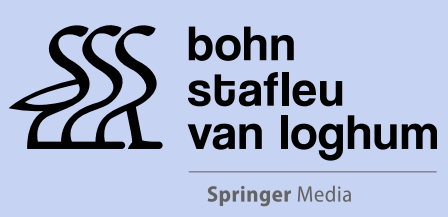

Houten 2021 
(volume and pressure) in addition to the physiological changes during pregnancy. This hypothesis is supported by the fact that we also found a decline in subpulmonary ventricular function later in pregnancy in women with elevated first-trimester NT-proBNP, whereas subpulmonary ventricular function remained stable in women with normal NT-ProBNP. Reduced subpulmonary ventricular function before and during pregnancy is associated with impaired uteroplacental circulation [13, 23, 24], which, in turn, is associated with adverse maternal and neonatal outcome [25, 26]. Therefore, close surveillance of subpulmonary ventricular function in women with elevated firsttrimester NT-proBNP may be warranted. Further studies are needed to determine whether the decline in subpulmonary ventricular function predicted by high NT-proBNP is only confined to pregnancy or persists thereafter.

Although the main focus of this study was on firsttrimester NT-proBNP, we also investigated the course of NT-proBNP throughout pregnancy. A difference in the course of NT-proBNP was found between women with and without CV complications. In line with the course of NT-proBNP in healthy pregnant women, NT-proBNP decreased in the second half of pregnancy in women without CV complications [18]. This decrease may reflect physiological cardiac adaptation to pregnancy. Conversely, NT-proBNP remained high in women who developed CV complications later in pregnancy. These data might be of additional value for the interpretation of trends in NT-proBNP during pregnancy in CHD women and may suggest that women with persisting elevated NT-proBNP might be at higher risk for $\mathrm{CV}$ complications due to cardiac maladaptation to pregnancy. Complication rates in our study were lower than those reported in previous studies [1, 4, 9, 27], which can possibly be explained by improved pre-pregnancy counselling and management of pregnancy of CHD women over the years [3].

\section{Limitations}

The number of CV complications in this study was relatively small, including mainly arrhythmias and no heart failure (worsening). These results are based on a specific heterogeneous study population and individual diseases may be underrepresented. NTproBNP levels differ per diagnosis and therefore these results might not be representative for each specific type of CHD [20]. Also, negative and positive predictive value are strongly dependent on the prevalence (and type) of CV complications and should be kept in mind when interpreting these results. Lastly, due to our population size and there being no controls available, we used a cut-off point for elevated firsttrimester NT-proBNP based on previous data from healthy pregnant women [18]. Larger studies are warranted to identify a cut-off point based on the occurrence of $\mathrm{CV}$ complications in pregnant CHD women.

\section{Conclusion}

In the current study, first-trimester NT-proBNP levels were associated with CV complications, particularly arrhythmias, and a decline in subpulmonary ventricular function later in pregnancy in women with CHD. Early NT-proBNP evaluation is useful for tailored care in pregnant women with CHD. NT-proBNP levels remain high throughout pregnancy in women that develop CV complications. In contrast, in women who do not develop CV complications, NT-proBNP levels decrease steadily from the second trimester onwards. Further studies are needed on the predictive value of NT-proBNP for subpulmonary ventricular deterioration during pregnancy in women with CHD.

Funding The ZAHARA III study was supported by a grant from ZonMW (91210050).

Conflict of interest A.S. Siegmund, P.G. Pieper, B.J. Bouma, F.M. Rosenberg, H. Groen, C.M. Bilardo, D.J. van Veldhuisen and M.G. Dickinson declare that they have no competing interests.

Open Access This article is licensed under a Creative Commons Attribution 4.0 International License, which permits use, sharing, adaptation, distribution and reproduction in any medium or format, as long as you give appropriate credit to the original author(s) and the source, provide a link to the Creative Commons licence, and indicate if changes were made. The images or other third party material in this article are included in the article's Creative Commons licence, unless indicated otherwise in a credit line to the material. If material is not included in the article's Creative Commons licence and your intended use is not permitted by statutory regulation or exceeds the permitted use, you will need to obtain permission directly from the copyright holder. To view a copy of this licence, visit http://creativecommons.org/licenses/by/4.0/.

\section{References}

1. Siu SC, Sermer M, Colman, et al. Prospective multicenter study of pregnancy outcomes in women with heart disease. Circulation. 2001;104:515-21.

2. Drenthen W, Boersma E, Balci A, et al. Predictors of pregnancy complications in women with congenital heart disease. Eur Heart J. 2010;31:2124-32.

3. Regitz-Zagrosek V, Roos-Hesselink JW, Bauersachs J, et al. 2018 ESC Guidelines for the management of cardiovascular diseases during pregnancy. Eur Heart J. 2018;39:3165-241.

4. Kampman MAM, Balci A, van Veldhuisen DJ, et al. N-terminal pro-B-type natriuretic peptide predicts cardiovascular complications in pregnant women with congenital heart disease. Eur Heart J. 2014;35:708-15.

5. Levin ER, Gardner DG, Samson WK. Natriuretic peptides. NEngl J Med. 1998;339:321-8.

6. Hunter S, Robson SC. Adaptation of the maternal heart in pregnancy. BrHeart J. 1992;68:540-3.

7. Kampman MAM, Balci A, Groen H, et al. Cardiac function and cardiac events 1-year postpartum in women with congenital heart disease. Am Heart J. 2015;169:298-304. 
8. Kampman MAM, Valente MA, van Melle JP, et al. Cardiac adaption during pregnancyin women with congenitalheart disease and healthy women. Heart. 2016;102:1302-8.

9. Drenthen W, Pieper PG, Roos-HesselinkJW, et al. Outcome of pregnancy in women with congenital heart disease: a literature review. JAm Coll Cardiol. 2007;49:2303-11.

10. Siegmund AS, Pieper PG, Mulder BJM, et al. Doppler gradients, valve area and ventricular function in pregnant women with aortic or pulmonary valve disease: left versus right. Int JCardiol. 2020;306:152-7.

11. Lemmer J, Heise G, Rentzsch A, et al. Right ventricular function ingrown-up patients after correction of congenital right heart disease. Clin Res Cardiol. 2011;1004:289-96.

12. Blyth KG, Groenning BA, Mark PB, et al. NT-proBNP can be used to detect right ventricular systolic dysfunction in pulmonary hypertension. Eur Respir J.2007;29:737-44.

13. Siegmund AS, Kampman MAM, Oudijk MA, et al. Maternal right ventricular function, uteroplacental circulation in first trimester, and pregnancy outcome in women with congenital heart disease. Ultrasound Obstet Gynecol. 2019;54:359-66.

14. Balci A, Sollie KM, Mulder BJM, et al. Associations between cardiovascular parameters and uteroplacental Doppler (blood) flow patterns during pregnancy in women with congenital heart disease: rationale and design of the Zwangerschap bij Aangeboren Hartafwijking (ZAHARA) II study. Am Heart J. 2011;161:269-75.

15. Baumgartner H, Hung J, Bermeijo J, et al. Echocardiographic assessment of valve stenosis: EAE/ASE recommendations for clinical practice. J Am Soc Echocardiogr. 2009;22:1-23.

16. Vahanian A, Alfieri O, Andreotti F, et al. Guidelines on the management of valvular heart disease (version 2012): the Joint Task Force on the Management of Valvular Heart Disease of theEuropean Society ofCardiology (ESC) and theEuropean Association for Cardio-Thoracic Surgery (EACTS). Eur JCardiothorac Surg. 2012;42:1-44.

17. Lang RM, Badano LP, Mor-Avi V, et al. Recommendations for cardiac chamber quantification by echocardiography in adults: an update from the American Society of Echocar- diography and the European Association of Cardiovascular Imaging. Eur Heart J Cardiovasc Imaging. 2015;16:233-70.

18. Franz MB, Andreas M, Schiessl B, et al. NT-proBNP is increased in healthy pregnancies compared to non-pregnant controls. Acta Obstet Gynecol Scand. 2009;88:234-7.

19. Ponikowski P, Voors AA, Anker SD, et al. 2016ESCGuidelines for the diagnosis and treatment of acute and chronic heart failure: the Task Force for the diagnosis and treatment of acute and chronic heart failure of the European Society of Cardiology (ESC) developed with the special contribution of the Heart Failure Association (HFA) of the ESC. Eur Heart J. 2016;37:2129-200.

20. Eindhoven JA, van den BoschAE, Ruys TPE, etal. N-terminal pro-B-type natriuretic peptide and its relationship with cardiac function in adults with congenital heart disease. JAm Coll Cardiol. 2013;62:1203-12.

21. Greutmann M, Pieper PG. Pregnancy in women with congenital heart disease. Eur Heart J. 2015;36:2491-9.

22. Walsh EP, Cecchin F. Arrhythmias in adult patients with congenital heart disease. Circulation. 2007;115:534-45.

23. Pieper PG, Balci A, Aarnoudse JG, et al. Uteroplacental blood flow, cardiac function, and pregnancy outcome in women with congenital heart disease. Circulation. 2013;128:2478-87.

24. Siegmund AS, Willems TP, Pieper PG, et al. Reduced right ventricularfunction on cardiovascular magnetic resonance imaging is associated with uteroplacental impairment in tetralogy of Fallot. JCardiovasc Magn Reson. 2020;22:52.

25. Brosens I, Pijnenborg R, Vercruysse L, et al. The "Great Obstetrical Syndromes" are associated with disorders of deep placentation. Am J Obstet Gynecol. 2011;204:193-201.

26. Aardema MW, Oosterhof H, Timmer A, et al. Uterine artery Doppler flow and uteroplacental vascular pathology in normal pregnancies and pregnancies complicated by preeclampsia and small for gestational age fetuses. Placenta. 2001;22:405-11.

27. Roos-Hesselink JW, Ruys TP, Stein JI, et al. Outcome of pregnancy in patients with structural or ischaemic heart disease: results of a registry of the European Society of Cardiology. Eur Heart J. 2013;34:657-65. 